\title{
TWO OSTROWSKI TYPE INEQUALITIES FOR THE STIELTJES INTEGRAL OF MONOTONIC FUNCTIONS
}

\author{
W.-S. Cheung and S.S. Dragomir
}

\begin{abstract}
Two integral inequalities of Ostrowski type for the Stieltjes integral are given. The first is for monotonic integrators and Hölder continuous integrands while the second considers the dual case, that is, for monotonic integrands and Hölder continuous integrators. Applications for the mid-point inequality that are useful in the numerical analysis of Stieltjes integrals are exhibited. Some connections with the generalised trapezoidal rule are also presented.
\end{abstract}

\section{INTRODUCTION}

In order to approximate the Stieltjes integral $\int_{a}^{b} p(x) d v(x)$, where $p, v:[a, b] \rightarrow \mathbb{R}$ are functions for which the above integral exists, Dragomir established in [9] the following integral identity:

$$
\begin{aligned}
{[u(b)-u(a)] f(x)-} & \int_{a}^{b} f(t) d u(t) \\
& =\int_{a}^{x}[u(t)-u(a)] d f(t)+\int_{x}^{b}[u(t)-u(b)] d f(t), \quad x \in[a, b]
\end{aligned}
$$

provided that the involved Stieltjes integrals exist. Earlier versions of (1.1) have been obtained in $[16,17,18]$. In the case $u(t)=t, t \in[a, b]$, the above identity reduces to the celebrated Montgomery identity (see [14, p. 565]) that has been extensively used by many authors in obtaining various inequalities of Ostrowski type. For a comprehensive recent collection of works, see the book $[12]$ and the papers $[1,2,3,4,5,13,15,19,20]$.

It has been shown in $[9]$ that, if $f:[a, b] \rightarrow \mathbb{R}$ is a function of bounded variation and $u:[a, b] \rightarrow \mathbb{R}$ is of $r$-H-Hölder type, that is,

$$
|u(x)-u(y)| \leqslant H|x-y|^{r} \text { for any } x, y \in[a, b],
$$

Received 23rd October, 2006

Research is supported in part by the Research Grants Council of the Hong Kong SAR, China (Project No. HKU7017/05P)

Copyright Clearance Centre, Inc. Serial-fee code: 0004-9727/07 \$A2.00+0.00. 
where $r \in(0,1]$ and $H>0$ are given, then

$$
\begin{aligned}
\mid[u(b)- & u(a)] f(x)-\int_{a}^{b} f(t) d u(t) \mid \\
& \leqslant H\left[(x-a)^{r} \bigvee_{a}^{x}(f)+(b-x)^{r} \bigvee_{x}^{b}(f)\right] \\
& \leqslant\left\{\begin{array}{l}
H\left[(x-a)^{r}+(b-x)^{r}\right]\left[\frac{1}{2} \bigvee_{a}^{b}(f)+\frac{1}{2}\left|\bigvee_{a}^{x}(f)-\bigvee_{x}^{b}(f)\right|\right] \\
H\left[(x-a)^{q r}+(b-x)^{q r}\right]^{1 / q}\left[\left(\bigvee_{a}^{x}(f)\right)^{p}+\left(\bigvee_{x}^{b}(f)\right)^{p}\right]^{1 / p} \\
H\left[\frac{1}{2}(b-a)+\left|x-\frac{a+b}{2}\right|\right]_{a}^{r} \bigvee_{a}^{b}(f) ;
\end{array}\right.
\end{aligned}
$$

for any $x \in[a, b]$, where $\bigvee_{c}^{d}(f)$ denotes the total variation of $f$ on $[c, d]$. Out of (1.3) we obtain the following mid-point inequality

$$
\left|[u(b)-u(a)] f\left(\frac{a+b}{2}\right)-\int_{a}^{b} f(t) d u(t)\right| \leqslant \frac{H(b-a)^{r}}{2^{r}} \cdot \bigvee_{a}^{b}(f)
$$

The dual result, that can be obtained on utilising a direct approach (see [10]), can be stated as follows:

If $u:[a, b] \rightarrow \mathbb{R}$ is of bounded variation on $[a, b]$ and $f:[a, b] \rightarrow \mathbb{R}$ is of $r$-H-Hölder type, then

$$
\left|[u(b)-u(a)] f(x)-\int_{a}^{b} f(t) d u(t)\right| \leqslant H\left[\frac{1}{2}(b-a)+\left|x-\frac{a+b}{2}\right|\right]^{r} \bigvee_{a}^{b}(u)
$$

for any $x \in[a, b]$. In particular, for $x=(a+b) / 2$, we get the mid-point inequality

$$
\left|[u(b)-u(a)] f\left(\frac{a+b}{2}\right)-\int_{a}^{b} f(t) d u(t)\right| \leqslant \frac{H(b-a)^{r}}{2^{r}} \cdot \bigvee_{a}^{b}(u) .
$$

The main aim of this paper is to continue the study that was begun in $[9,10]$ by considering other classes of functions for which the error bound in approximating the Stieltjes integral $\int_{a}^{b} f(t) d u(t)$ by the quantity $[u(b)-u(a)] f(x)$ with $x \in[a, b]$ can easily be computed a priori. Applications for the mid-point and generalised trapezoidal rules are also given. 


\section{The Case of Monotonic Integrators}

The following result may be stated.

THEOREM 1. Let $f:[a, b] \rightarrow \mathbb{R}$ be a function of $r$-H-Hölder type with $r \in(0,1]$ and $H>0$, and $u:[a, b] \rightarrow \mathbb{R}$ be a monotonic nondecreasing function on $[a, b]$. Then

$$
\begin{aligned}
& \left|[u(b)-u(a)] f(x)-\int_{a}^{b} f(t) d u(t)\right| \\
& \leqslant H\left[(b-x)^{r} u(b)-(x-a)^{r} u(a)\right. \\
& \left.\quad+r\left\{\int_{a}^{x} \frac{u(t)}{(x-t)^{1-r}} d t-\int_{x}^{b} \frac{u(t)}{(t-x)^{1-r}} d t\right\}\right] \\
& \leqslant H\left\{(b-x)^{r}[u(b)-u(x)]+(x-a)^{r}[u(x)-u(a)]\right\} \\
& \leqslant H\left[\frac{1}{2}(b-a)+\left|x-\frac{a+b}{2}\right|\right]^{r}[u(b)-u(a)],
\end{aligned}
$$

for any $x \in[a, b]$.

Proof: First of all we remark that if $p:[a, b] \rightarrow \mathbb{R}$ is continuous and $v:[a, b] \rightarrow \mathbb{R}$ is monotonic nondecreasing, then the Stieltjes integral $\int_{a}^{b} p(t) d v(t)$ exists and:

$$
\left|\int_{a}^{b} p(t) d v(t)\right| \leqslant \int_{a}^{b}|p(t)| d v(t)
$$

Making use of this property and the fact that $f$ is of $r$-H-Hölder type, we can state that

$$
\begin{aligned}
\left|[u(b)-u(a)] f(x)-\int_{a}^{b} f(t) d u(t)\right| & =\left|\int_{a}^{b}[f(x)-f(t)] d u(t)\right| \\
& \leqslant \int_{a}^{b}|f(x)-f(t)| d u(t) \\
& \leqslant H \int_{a}^{b}|x-t|^{\tau} d u(t) .
\end{aligned}
$$

By the integration by parts formula for the Stieltjes integral we have

$$
\begin{aligned}
\int_{a}^{b} \mid & -\left.t\right|^{r} d u(t) \\
& =\int_{a}^{x}(x-t)^{r} d u(t)+\int_{x}^{b}(t-x)^{r} d u(t) \\
& =\left.(x-t)^{r} u(t)\right|_{a} ^{x}+r \int_{a}^{x} \frac{u(t)}{(x-t)^{1-r}} d t+\left.(t-x)^{r} u(t)\right|_{x} ^{b}-r \int_{x}^{b} \frac{u(t)}{(t-x)^{1-r}} d t \\
& =(b-x)^{r} u(b)-(x-a)^{r} u(a)+r\left[\int_{a}^{x} \frac{u(t)}{(x-t)^{1-r}} d t-\int_{x}^{b} \frac{u(t)}{(t-x)^{1-r}} d t\right]
\end{aligned}
$$


which together with (2.3) proves the first inequality in (2.1).

Now, by the monotonicity property of $u$ we have

$$
\int_{a}^{x} \frac{u(t) d t}{(x-t)^{1-r}} \leqslant u(x) \int_{a}^{x} \frac{d t}{(x-t)^{1-r}}=\frac{(x-a)^{r} u(x)}{r}
$$

and

$$
\int_{x}^{b} \frac{u(t) d t}{(t-x)^{1-r}} \geqslant u(x) \int_{x}^{b} \frac{d t}{(t-x)^{1-r}}=\frac{(b-x)^{r} u(x)}{r}
$$

giving that

$$
\int_{a}^{x} \frac{u(t) d t}{(x-a)^{1-r}}-\int_{x}^{b} \frac{u(t) d t}{(t-x)^{1-r}} \leqslant \frac{1}{r}\left[(x-a)^{r} u(x)-(b-x)^{r} u(x)\right] .
$$

This inequality implies that

$$
\begin{aligned}
(b-x)^{r} u(b)-(x-a)^{r} u(a)+r\left[\int_{a}^{x} \frac{u(t)}{(x-t)^{1-r}} d t-\int_{x}^{b} \frac{u(t)}{(t-x)^{1-r}} d t\right] \\
\quad \leqslant(b-x)^{r} u(b)-(x-a)^{r} u(a)+(x-a)^{r} u(x)-(b-x)^{r} u(x) \\
=(b-x)^{r}[u(b)-u(x)]+(x-a)^{r}[u(x)-u(a)]
\end{aligned}
$$

and the second part of inequality (2.1) is also proved.

The last part is obvious by the property of max function and we omit the details here.

REMARK 1. If $f$ is assumed to be $L$-Lipschitzian, that is,

$$
|f(x)-f(y)| \leqslant L|x-y| \text { for any } x, y \in[a, b],
$$

where $L>0$ is given, then for $u:[a, b] \rightarrow \mathbb{R}$ being monotonic nondecreasing on $[a, b]$ the inequality (2.1) will produce the simple result:

$$
\begin{aligned}
\mid[u(b) & -u(a)] f(x)-\int_{a}^{b} f(t) d u(t) \mid \\
& \leqslant L\left[b u(b)+a u(a)-x[u(a)+u(b)]+\int_{a}^{b} \operatorname{sgn}(x-t) u(t) d t\right] \\
& \leqslant L[(b-x)[u(b)-u(x)]+(x-a)[u(x)-u(a)]] \\
& \leqslant L\left[\frac{1}{2}(b-a)+\left|x-\frac{a+b}{2}\right|\right][u(b)-u(a)]
\end{aligned}
$$

for any $x \in[a, b]$.

A particular case that may be useful in applications is the following mid-point type inequality. 
COROLLARY 1. With the assumptions in Theorem 1, we have:

$$
\begin{aligned}
& \left|[u(b)-u(a)] f\left(\frac{a+b}{2}\right)-\int_{a}^{b} f(t) d u(t)\right| \\
& \leqslant H\left[\frac{(b-a)^{r}}{2^{r}}[u(b)-u(a)]\right. \\
& \left.\quad+r\left\{\int_{a}^{(a+b) / 2} \frac{u(t) d t}{((a+b) / 2-t)^{1-r}}-\int_{(a+b) / 2}^{b} \frac{u(t) d t}{(t-(a+b) / 2)^{1-r}}\right\}\right] \\
& \quad \leqslant \frac{H(b-a)^{r}}{2^{r}}[u(b)-u(a)] .
\end{aligned}
$$

In particular, if $f$ is a $L$-Lipschitzian function, we have

$$
\begin{aligned}
\mid[u(b)- & u(a)] f\left(\frac{a+b}{2}\right)-\int_{a}^{b} f(t) d u(t) \mid \\
& \leqslant L\left[\frac{(b-a)[u(b)-u(a)]}{2}+\int_{a}^{b} \operatorname{sgn}\left(\frac{a+b}{2}-t\right) u(t) d t\right] \\
& \leqslant \frac{L \cdot(b-a)}{2}[u(b)-u(a)] .
\end{aligned}
$$

REMARK 2. We observe that the first inequality in (2.9) is sharp. Indeed, if we choose $f, u:[a, b] \rightarrow \mathbb{R}, f(t)=|t-(a+b) / 2|, u(t)=t-(a+b) / 2$, we notice that $f$ is $L$ Lipschitzian with the constant $L=1$ and $u$ is monotonic nondecreasing on $[a, b]$. Also:

$$
\begin{aligned}
& {[u(b)-u(a)] f\left(\frac{a+b}{2}\right)-\int_{a}^{b} f(t) d t=-\int_{a}^{b}\left|t-\frac{a+b}{2}\right| d t=-\frac{(b-a)^{2}}{4}} \\
& \begin{aligned}
\frac{(b-a)[u(b)-u(a)]}{2}+\int_{a}^{b} \operatorname{sgn}\left(\frac{a+b}{2}-t\right) u(t) d t \\
\quad=\frac{(b-a)^{2}}{2}-\int_{a}^{b}\left|t-\frac{a+b}{2}\right| d t=\frac{(b-a)^{2}}{4},
\end{aligned}
\end{aligned}
$$

which shows that in both sides of $(2.9)$ we have the same quantity $(b-a)^{2} / 4$.

REMARK 3. In terms of probability density functions, if $w:[a, b] \rightarrow[0, \infty)$ is such that $\int_{a}^{b} w(s) d s=1$, then writing out the inequality (2.1) for $u(t)=W(t):=\int_{a}^{t} w(s) d s$, we obtain:

$$
\begin{aligned}
\mid f(x)- & \int_{a}^{b} w(s) f(s) d s \mid \\
& \leqslant H\left[(b-x)^{r}+r\left\{\int_{a}^{x} \frac{W(t)}{(x-t)^{1-r}} d t-\int_{x}^{b} \frac{W(t)}{(t-x)^{1-r}} d t\right\}\right] \\
& \leqslant H\left[(b-x)^{r} \int_{x}^{b} w(s) d s+(x-a)^{r} \int_{a}^{x} w(s) d s\right] \\
& \leqslant H\left[\frac{1}{2}(b-a)+\left|x-\frac{a+b}{2}\right|\right]^{r}
\end{aligned}
$$


for any $x \in[a, b]$, where, as above, $f$ is of $r$-H-Hölder type.

The Lipschitzian case provides the simpler inequality:

$$
\begin{aligned}
\left|f(x)-\int_{a}^{b} w(s) f(s) d s\right| & \leqslant L\left[b-x+\int_{a}^{b} \operatorname{sgn}(x-t) W(t) d t\right] \\
& \leqslant L\left[\frac{1}{2}(b-a)+\left|x-\frac{a+b}{2}\right|\right]
\end{aligned}
$$

for any $x \in[a, b]$.

Finally, the weighted trapezoidal inequality for Hölder continuous functions reads as

$$
\begin{aligned}
& \left|f\left(\frac{a+b}{2}\right)-\int_{a}^{b} w(s) f(s) d s\right| \\
& \quad \leqslant H\left[\frac{(b-a)^{r}}{2^{r}}+r\left\{\int_{a}^{(a+b) / 2} \frac{W(t)}{((a+b) / 2-t)^{1-r}} d t-\int_{(a+b) / 2}^{b} \frac{W(t) d t}{(t-(a+b) / 2)^{1-r}}\right\}\right] \\
& \quad \leqslant \frac{H(b-a)^{r}}{2^{r}}
\end{aligned}
$$

while for Lipschitzian functions it will have the form

$$
\begin{aligned}
\mid f\left(\frac{a+b}{2}\right)-\int_{a}^{b} w(s) & f(s) d s \mid \\
& \leqslant L \cdot\left[\frac{b-a}{2}+\int_{a}^{b} \operatorname{sgn}\left(\frac{a+b}{2}-t\right) W(t) d t\right] \leqslant \frac{1}{2} L(b-a) .
\end{aligned}
$$

The uniform distribution $w(s)=1 /(b-a), s \in[a, b]$, will then provide the following inequality:

$$
\begin{aligned}
\mid f(x)- & \frac{1}{b-a} \int_{a}^{b} f(t) d t \mid \\
& \leqslant H\left[(b-x)^{r}+\frac{r}{b-a}\left\{\int_{a}^{x} \frac{(t-a)}{(x-t)^{1-r}} d t\right.\right. \\
& \left.\left.\quad-\int_{x}^{b} \frac{(t-a)}{(t-x)^{1-r}} d t\right\}\right](=: H T, \text { say }) \\
& \leqslant \frac{H}{b-a}\left[(b-x)^{r+1}+(x-a)^{r+1}\right] \leqslant H\left[\frac{1}{2}(b-a)+\left|x-\frac{a+b}{2}\right|\right]^{r} .
\end{aligned}
$$

Since

$$
\begin{aligned}
\int_{a}^{x} \frac{(t-a)}{(x-t)^{1-r}} d t & =\int_{a}^{x}(t-a)(x-t)^{r-1} d t \\
& =(x-a)^{r+1} \int_{0}^{1} s(1-s)^{r-1} d s \\
& =(x-a)^{r+1} B(2, r)=\frac{(x-a)^{r+1}}{r(r+1)}
\end{aligned}
$$


where $B(p, q):=\int_{0}^{1} s^{p-1}(1-s)^{q-1} d s, p, q>0$ is the Euler's Beta function, and

$$
\begin{aligned}
\int_{x}^{b} \frac{(t-a)}{(t-x)^{1-r}} d t & =\int_{x}^{b} \frac{t-b+b-a}{(t-x)^{1-r}} d t \\
& =(b-a) \int_{x}^{b} \frac{d t}{(t-x)^{1-r}}-\int_{x}^{b} \frac{(b-t) d t}{(t-x)^{1-r}} \\
& =(b-a) \cdot \frac{(b-x)^{r}}{r}-\int_{x}^{b}(b-t)(t-x)^{r-1} d t \\
& =(b-a) \cdot \frac{(b-x)^{r}}{r}-(b-x)^{r+1} \int_{0}^{1} s(1-s)^{r-1} d s \\
& =(b-a) \cdot \frac{(b-x)^{r}}{r}-(b-x)^{r+1} B(2, r) \\
& =\frac{(b-a)(b-x)^{r}}{r}-\frac{(b-x)^{r+1}}{r(r+1)}
\end{aligned}
$$

hence $T$, defined above, has the form

$$
\begin{aligned}
T & =(b-x)^{r}+\frac{r}{b-a}\left\{\frac{(x-a)^{r+1}}{r(r+1)}-\frac{(b-a)(b-x)^{r}}{r}+\frac{(b-x)^{r+1}}{r(r+1)}\right\} \\
& =\frac{(x-a)^{r+1}+(b-\alpha)^{r+1}}{r+1} .
\end{aligned}
$$

Therefore, from the first inequality in (2.14) we deduce

$$
\left|f(x)-\frac{1}{b-a} \int_{a}^{b} f(t) d t\right| \leqslant \frac{H}{r+1}\left[\left(\frac{x-a}{b-a}\right)^{r+1}+\left(\frac{b-x}{b-x}\right)^{r+1}\right](b-a)^{r}
$$

for any $x \in[a, b]$, which has been obtained before (see for instance $[8, \mathbf{8}]$ ).

\section{ThE CASE OF MONOTONIC INTEGRANDS}

It is natural now to investigate the dual case, that is, where the integrand $f$ is assumed to be monotonic nondecreasing while the integrator $u$ is Hölder continuous.

TheOREM 2. Let $f:[a, b] \rightarrow \mathbb{R}$ be monotonic nondecreasing on $[a, b]$ and $u$ : $[a, b] \rightarrow \mathbb{R}$ of $r$-H-Hölder type. Then

$$
\begin{aligned}
\mid[u(b)- & u(a)] f(x)-\int_{a}^{b} f(t) d u(t) \mid \\
& \leqslant H\left[\left[(x-a)^{r}-(b-a)^{r}\right] f(x)+r\left\{\int_{x}^{b} \frac{f(t) d t}{(b-t)^{1-r}}-\int_{a}^{x} \frac{f(t) d t}{(t-a)^{1-r}}\right\}\right] \\
& \leqslant H\left\{(b-x)^{r}[f(b)-f(x)]+(x-a)^{r}[f(x)-f(a)]\right\} \\
& \leqslant H\left[\frac{1}{2}(b-a)+\left|x-\frac{a+b}{2}\right|\right]^{r}[f(b)-f(a)] .
\end{aligned}
$$


Proof: Utilising the integral identity (1.1) and the hypothesis, we have successively

$$
\begin{aligned}
\mid[u(b) & -u(a)] f(x)-\int_{a}^{b} f(t) d u(t) \mid \\
& \leqslant \int_{a}^{x}|u(t)-u(a)| d f(t)+\int_{x}^{b}|u(s)-u(t)| d f(t) \\
& \leqslant H\left[\int_{a}^{x}(t-a)^{r} d f(t)+\int_{x}^{b}(b-t)^{r} d f(t)\right] \\
& =H\left[\left.(t-a)^{r} f(t)\right|_{a} ^{x}-r \int_{a}^{x} \frac{f(t) d t}{(t-a)^{1-r}}+\left.(b-t)^{r} f(t)\right|_{x} ^{b}+r \int_{x}^{b} \frac{f(t) d t}{(b-t)^{1-r}}\right] \\
& =H\left[(x-a)^{r} f(x)-(b-x)^{r} f(x)+r\left\{\int_{x}^{b} \frac{f(t) d t}{(b-t)^{1-r}}-\int_{a}^{x} \frac{f(t) d t}{(t-a)^{1-r}}\right\}\right]
\end{aligned}
$$

proving the first inequality in (3.1).

Since $f$ is monotonic nondecreasing on $[a, b]$, hence

$$
\int_{x}^{b} \frac{f(t) d t}{(b-t)^{1-r}} \leqslant f(b) \int_{x}^{b} \frac{d t}{(b-t)^{1-r}}=\frac{f(b)(b-x)^{r}}{r}
$$

and

giving that

$$
\int_{a}^{x} \frac{f(t) d t}{(t-a)^{1-r}} \geqslant f(a) \int_{a}^{x} \frac{d t}{(t-a)^{1-r}}=\frac{f(a)(x-a)^{r}}{r}
$$

$$
\int_{x}^{b} \frac{f(t) d t}{(b-t)^{1-r}}-\int_{a}^{x} \frac{f(t) d t}{(t-a)^{1-r}} \leqslant \frac{1}{r}\left[f(b)(b-x)^{r}-f(a)(x-a)^{r}\right]
$$

which obviously implies that

$$
\begin{aligned}
(x-a)^{r} f(x)- & (b-x)^{r} f(x)+r\left[\int_{x}^{b} \frac{f(t) d t}{(b-t)^{1-r}}-\int_{a}^{x} \frac{f(t) d t}{(t-a)^{1-r}}\right] \\
& \leqslant(x-a)^{r} f(x)-(b-x)^{r} f(x)+f(b)(b-x)^{r}-f(a)(x-a)^{r} \\
& =(b-x)^{r}[f(b)-f(x)]+(x-a)^{r}[f(x)-f(a)],
\end{aligned}
$$

which together with (3.2) provides the second inequality in (3.1).

The last inequality is obvious, since

$$
\begin{aligned}
(b-x)^{r}[f(b)-f(x)]+(x-a)^{r}[f(x)-f(a)] \\
\leqslant \max \left\{(b-x)^{r},(x-a)^{r}\right\}[f(b)-f(a)] \\
=[\max \{b-x, x-a\}]^{r}[f(b)-f(a)] \\
=\left[\frac{1}{2}(b-a)+\left|x-\frac{a+b}{2}\right|\right]^{r}[f(b)-f(a)],
\end{aligned}
$$

for any $x \in[a, b]$. 
REMARK 4. The particular case of $L$-Lipschitzian functions provides a much simpler result:

$$
\begin{aligned}
\mid[u(b)-u(a)] f(x) & -\int_{a}^{b} f(t) d u(t) \mid \\
& \leqslant L\left[(2 x-a-b) f(x)+\int_{a}^{b} \operatorname{sgn}(t-x) f(t) d t\right] \\
& \leqslant L\{(b-x)[f(b)-f(x)]+(x-a)[f(x)-f(a)]\} \\
& \leqslant L\left[\frac{1}{2}(b-a)+\left|x-\frac{a+b}{2}\right|\right][f(b)-f(a)]
\end{aligned}
$$

for any $x \in[a, b]$.

A particular case that can be useful in applications is the following one.

COROLlary 2. With the assumptions in Theorem 2 we have:

$$
\begin{aligned}
\mid[u(b)-u(a)] & f\left(\frac{a+b}{2}\right)-\int_{a}^{b} f(t) d u(t) \mid \\
& \leqslant r H\left\{\int_{(a+b) / 2}^{b} \frac{f(t) d t}{(b-t)^{1-r}}-\int_{a}^{(a+b) / 2} \frac{f(t) d t}{(t-a)^{1-r}}\right\} \\
& \leqslant \frac{H(b-a)^{r}}{2^{r}}[f(b)-f(a)] .
\end{aligned}
$$

In particular, for $u$ a $L$-Lipschitizian function, we have

$$
\begin{aligned}
\mid[u(b)-u(a)] f\left(\frac{a+b}{2}\right)- & \int_{a}^{b} f(t) d u(t) \mid \\
& \leqslant L \int_{a}^{b} \operatorname{sgn}\left(t-\frac{a+b}{2}\right) \cdot f(t) d t \leqslant \frac{1}{2} L[f(b)-f(a)] .
\end{aligned}
$$

A much simpler result follows:

$$
\begin{aligned}
\mid[u(b)- & u(a)] f(x)-\int_{a}^{b} f(t) d u(t) \mid \\
& \leqslant L\left[(2 x-a-b) f(x)+\int_{a}^{b} \operatorname{sgn}(t-x) f(t) d t\right] \\
& \leqslant L\{(b-x)[f(b)-f(x)]+(x-a)[f(x)-f(a)]\} \\
& \leqslant L\left[\frac{1}{2}(b-a)+\left|x-\frac{a+b}{2}\right|\right][f(b)-f(a)]
\end{aligned}
$$

for any $x \in[a, b]$.

REMARK 5. The inequalities (3.5) are sharp. Indeed, if we take $u, f:[a, b] \rightarrow \mathbb{R}$, $u(t)=|t-(a+b) / 2|$ and $f(t)=\operatorname{sgn}(t-(a+b) / 2)$, then $u$ is $L$-Lipschitzian with $L=1$ 
and $f$ is monotonic nondecreasing on $[a, b]$. Also,

$$
\begin{aligned}
{[u(b)-u(a)] } & f\left(\frac{a+b}{2}\right)-\int_{a}^{b} f(t) d u(t) \\
& =-\left[\int_{a}^{(a+b) / 2}(-1) \cdot d\left(\frac{a+b}{2}-t\right)+\int_{(a+b) / 2}^{b}(+1) \cdot d t\left(t-\frac{a+b}{2}\right)\right] \\
& =-(b-a),
\end{aligned}
$$

and

$$
\int_{a}^{b} \operatorname{sgn}\left(t-\frac{a+b}{2}\right) f(t) d t=b-a
$$

and then we get in all sides of the inequality (3.5) the same quantity $(b-a)$.

REMARK 6. In the case when $u(t)=t, t \in[a, b]$, out of (3.6) we deduce the Ostrowski type inequality:

$$
\begin{aligned}
\left|f(x)-\frac{1}{b-a} \int_{a}^{b} f(t) d t\right| & \leqslant \frac{1}{b-a}\left[[2 x-(a+b)]+\int_{a}^{b} \operatorname{sgn}(t-x) f(t) d t\right] \\
& \leqslant \frac{1}{b-a}\{(b-x)[f(b)-f(x)]+(x-a)[f(x)-f(a)]\} \\
& \leqslant\left[\frac{1}{2}+\frac{|x-(a+b) / 2|}{b-a}\right][f(b)-f(a)]
\end{aligned}
$$

that has been obtained in [11] (see also [12]).

\section{Some Results for a Generalised Trapezoidal Rule}

In [7], the authors have considered the following generalised trapezoidal formula:

$$
[u(b)-u(x)] f(b)+[u(x)-u(a)] f(a), \quad x \in[a, b]
$$

to approximate the Stieltjes integral $\int_{a}^{b} f(t) d u(t)$. They proved the inequality

$$
\begin{aligned}
& \left|\int_{a}^{b} f(t) d u(t)-[u(b)-u(x)] f(a)-[u(x)-u(a)] f(a)\right| \\
& \leqslant H\left[\frac{1}{2}(b-a)+\left|x-\frac{a+b}{2}\right|\right]_{a}^{r} \bigvee_{a}^{b}(f)
\end{aligned}
$$

for any $x \in[a, b]$, provided that $f:[a, b] \rightarrow \mathbb{R}$ is of bounded variation on $[a, b]$ and $u$ is of $r$ - $H$-Hölder type.

The best inequality one can obtain from (4.1) is the following:

$$
\begin{aligned}
\left|\int_{a}^{b} f(t) d u(t)-\left[u(b)-u\left(\frac{a+b}{2}\right)\right] f(a)-\left[u\left(\frac{a+b}{2}\right)-u(a)\right] f(a)\right| & \\
& \leqslant \frac{H(b-a)}{2^{r}} \bigvee_{a}^{b}(f) .
\end{aligned}
$$


We observe that if $p, v:[a, b] \rightarrow \mathbb{R}$ are a pair of functions for which the Stieltjes integral $\int_{a}^{b} p(t) d v(t)$ exists, then, on application of the integration by parts formula, we have

$$
\begin{aligned}
{[v(b)-v(a)] } & p(x)-\int_{a}^{b} p(t) d v(t) \\
= & {[v(b)-v(a)] p(x)-\left[p(b) v(b)-p(a) v(a)-\int_{a}^{b} v(t) d p(t)\right] } \\
& =\int_{a}^{b} v(t) d p(t)-v(a)[p(x)-p(a)]-v(b)[p(b)-p(x)]
\end{aligned}
$$

Therefore, any inequality of Ostrowski type for the difference

$$
[v(b)-v(a)] p(x)-\int_{a}^{b} p(t) d v(t)
$$

would give a corresponding inequality for the generalised trapezoidal approximation of the dual Stieltjes integral:

$$
\int_{a}^{b} v(t) d p(t)-v(a)[p(x)-p(a)]-v(b)[p(b)-p(x)]
$$

If $v$ is of $r$-H-Hölder type and $p$ is of bounded variation, then by (1.3) and (4.3) we recapture the result from $[6]$ :

$$
\begin{aligned}
\mid \int_{a}^{b} v(t) d p(t) & -v(a)[p(x)-p(a)]-v(b)[p(b)-p(x)] \mid \\
& \leqslant H\left[(x-a)^{r} \bigvee_{a}^{x}(p)+(b-x)^{r} \bigvee_{x}^{b}(p)\right] \\
& \leqslant\left\{\begin{aligned}
H\left[(x-a)^{r}+(b-x)^{r}\right]\left[\frac{1}{2} \bigvee_{a}^{b}(p)+\frac{1}{2}\left|\bigvee_{a}^{x}(p)-\bigvee_{x}^{b}(p)\right|\right] \\
\left.H\left[(x-a)^{q r}+(b-x)^{q r}\right]^{1 / q}\left[\prod_{a}^{x}(p)\right]^{p}+\left[\bigvee_{x}^{b}(p)\right]^{p}\right]^{1 / p} \\
\text { if } p>1, \frac{1}{p}+\frac{1}{q}=1 ; \\
H\left[\frac{1}{2}(b-a)+\left|x-\frac{a+b}{2}\right|\right]_{a}^{r} \bigsqcup_{a}^{b}(p)
\end{aligned}\right.
\end{aligned}
$$

for $x \in[a, b]$.

If we use (1.5) and the identity (4.3) above, then we can get the result in (4.1).

Now, if $p$ is of $r-H$-Hölder type and $v$ is monotonic nondecreasing, then by Theorem $I$ and (4.3) we have the inequality

$$
\left|\int_{a}^{b} v(t) d p(t)-v(a)[p(x)-p(a)]-v(b)[p(b)-p(x)]\right|
$$




$$
\begin{aligned}
& \leqslant H\left\{(b-x)^{r} v(b)-(x-a)^{r} v(a)\right. \\
& \left.\quad+r\left\{\int_{a}^{x} \frac{v(t)}{(x-t)^{1-r}} d t-\int_{x}^{b} \frac{v(t)}{(t-x)^{1-r}} d t\right\}\right\} \\
& \leqslant H\left\{(b-x)^{r}[v(b)-v(x)]+(x-a)^{r}[v(x)-v(a)]\right\} \\
& \leqslant H\left[\frac{1}{2}(b-a)+\left|x-\frac{a+b}{2}\right|\right]^{r}[v(b)-v(a)]
\end{aligned}
$$

for any $x \in[a, b]$.

Finally, by employing Theorem 2 and the identity (4.3), we can state that for $p$ monotonic nondecreasing and $v$ of $r$ - $H$-Hölder type, we have:

$$
\begin{aligned}
\mid \int_{a}^{b} v(t) & d p(t)-v(a)[p(x)-p(a)]-v(b)[p(b)-p(x)] \mid \\
\leqslant & H\left[\left[(x-a)^{r}-(b-x)^{r}\right] p(x)+r\left\{\int_{x}^{b} \frac{p(t) d t}{(b-t)^{1-r}}-\int_{a}^{x} \frac{p(t) d t}{(t-a)^{1-r}}\right\}\right] \\
\leqslant & \leqslant\left[(b-x)^{r}[p(b)-p(x)]+(x-a)^{r}[p(x)-p(a)]\right] \\
\leqslant & \leqslant\left[\frac{1}{2}(b-a)+\left|x-\frac{a+b}{2}\right|\right]^{r}[p(b)-p(a)]
\end{aligned}
$$

for each $x \in[a, b]$.

\section{REFERENCES}

[1] A.G. Anastassiou, 'Univariate Ostrowski inequalities, revisited', Monatsh. Math. 135 (2002), 175-189.

[2] A. Aglić-Aljinović and J. Pečarić, 'On some Ostrowski type inequalities via Montgomery identity and Taylor's formula', Tamkang J. Math. 36 (2005), 199-218.

[3] A. Aglić-Aljinović, J. Pečarić and A. Vukelić, 'On some Ostrowski type inequalities via Montgomery identity and Taylor's formula II', Tamkang J. Math. 36 (2005), 279-301.

[4] P. Cerone, 'Approximate multidimensional integration through dimension reduction via the Ostrowski functional', Nonlinear Funct. Anal. Appl. 8 (2003), 313-333.

[5] P. Cerone and S.S. Dragomir, 'On some inequalities arising from Montgomery's identity', J. Comput. Anal. Appl. 5 (2003), 341-367.

[6] P. Cerone and S.S. Dragomir, 'New bounds for the three-point rule involving the Riemann-Stieltjes integral', in Advances in Statistics, Combinatorics and Related Areas (World Sci. Publishing, River Edge, N.J., 2002), pp. 53-62.

[7] S.S. Dragomir, C. Buşe, M.V. Boldea and L. Brăescu, 'A generalisation of the trapezoidal rule for the Riemann-Stieltjes integral and applications', Nonlin. Anal. Forum 6 (2001), 337-351.

[8] S.S. Dragomir, P. Cerone, J. Roumeliotis and S. Wang, 'A weighted version of Ostrowski inequality for mappings of Hölder type and applications in numerical analysis', Bull. Math. Soc. Sci. Math. R.S. Roumanie (N.S.) 42 (1999), 301-314. 
[9] S.S. Dragomir, 'On the Ostrowski's inequality for Riemann-Stieltjes integral', Korean $J$. Appl. Math. 7 (2000), 477-485.

[10] S.S. Dragomir, 'On the Ostrowski inequality for Riemann-Stieltjes integral $\int_{a}^{b} f(t) d u(t)$ where $f$ is of Hölder type and $u$ is of bounded variation and applications', J. KSIAM 5 (2001), 35-45.

[11] S.S. Dragomir, 'Ostrowski's inequality for montonous mappings and applications', $J$. KSIAM 3 (1999), 127-135.

[12] S.S. Dragomir and Th.M. Rassias (Eds.), Ostrowski type inequalities and applications in numerical integration (Kluwer Academic Publishers, Dordrecht, 2002).

[13] P. Kumar, 'The Ostrowski type moment integral inequalities and moment-bounds for continuous random variables', Comput. Math. Appl. 49 (2005), 1929-1940.

[14] D.S. Mitrinović, J.E. Pečarić and A.M. Fink, Inequalities involving functions and their integrals and derivatives (Kluwer Academic Publishers, Dordrecht, 1991).

[15] B.G. Pachpatte, 'A note on Ostrowski like inequalities', J. Inequal. Pure Appl. Math. 6 (2005), 4.

[16] J.E. Pečarić, 'On the Čebyšev inequality', Bul. Ştiinţ. Tehn. Inst. Politehn. "Traian Vuia" Timişoara 25 (39) (1981), 5-9.

[17] J.E. Pečarić, 'A new proof of the Jensen-Steffensen inequality', Mathematica (Cluj) 23 (1981), 73-77.

[18] J.E. Pečarić, 'On the Ostrowski generalization of Čebyšev's inequality', J. Math. Anal. Appl. 102 (1984), 479-487.

[19] A. Sofo, 'Integral inequalities for $N$-times differentiable mappings', in Ostrowski type inequalities and applications in numerical integration (Kluwer Acad. Publ., Dordrecht, 2002), pp. 65-139.

[20] N. Ujević, 'Sharp inequalities of Simpson type and Ostrowski type', Comput. Math. Appl. 48 (2004), 145-151.

Department of Mathematics

University of Hong Kong

Hong Kong

e-mail: wscheung@hku.hk
School of Computer Science and Mathematics Victoria University

PO Box 14428, MCMC 8001

Victoria, Australia

e-mail: sever@csm.vu.edu.au 